\title{
Reviewers of the Special Issue: Molecules to Behavior (May 2011)
}

The Editors of the special issue would like to thank the following reviewers:

Ann Swillen (Belgium)

Chris McManus (UK)

Stewart Einfeld (Australia)

Cornel Popovici (France)

Anna Byars (USA)

Robert Waltereit (Germany)

Henri Bléhaut (France)

Robert Feldmann (Germany)

Sylvie Tordjman (France)

Mustafa Sahin (USA)

Carrie Bearden (USA)

Deborah McCartney (UK)

Richard Simensen (USA)

Peter Lockhart (New Zealand)

Christopher J Howe (UK)
Annette Karmiloff-Smith (UK)

François-Xavier Alario (France)

François-Xavier Coude (France)

Bruno Gepner (France)

Jean-Christophe Cassel (France)

Pamela Bryden (Canada)

Jacqueline Fagard (France)

Flora Tassone (USA)

Vicky Whittemore (USA)

David Skuse, (UK)

Michael Brammer (UK)

Carl Stafstrom (USA)

Gene Fisch (USA)

James Harris (USA) 